\title{
Phase Transition of Black Holes in Brans-Dicke Born-Infeld Gravity through Geometrical Thermodynamics
}

\author{
S. H. Hendi, ${ }^{1,2}$ M. S. Talezadeh, ${ }^{1}$ and Z. Armanfard ${ }^{1}$ \\ ${ }^{1}$ Physics Department and Biruni Observatory, College of Sciences, Shiraz University, Shiraz 71454, Iran \\ ${ }^{2}$ Research Institute for Astronomy and Astrophysics of Maragha (RIAAM), P.O. Box 55134-441, Maragha, Iran \\ Correspondence should be addressed to S. H. Hendi; hendi@shirazu.ac.ir
}

Received 10 March 2017; Accepted 13 July 2017; Published 11 September 2017

Academic Editor: Giovanni Amelino-Camelia

Copyright (c) 2017 S. H. Hendi et al. This is an open access article distributed under the Creative Commons Attribution License, which permits unrestricted use, distribution, and reproduction in any medium, provided the original work is properly cited. The publication of this article was funded by SCOAP ${ }^{3}$.

Using the geometrical thermodynamic approach, we study phase transition of Brans-Dicke Born-Infeld black holes. We apply introduced methods and describe their shortcomings. We also use the recently proposed new method and compare its results with those of canonical ensemble. By considering the new method, we find that its Ricci scalar diverges in the places of phase transition and bound points. We also show that the bound point can be distinguished from the phase transition points through the sign of thermodynamical Ricci scalar around its divergencies.

\section{Introduction}

General relativity is accepted as a standard theory of gravitation and is able to pass more observational tests [1]. Although this theory is successful in various domains, it cannot describe some experimental evidences such as the accelerating expansion of the Universe [2-4]. Moreover, the general relativity theory is not consistent with Mach's principle nor Dirac's large number hypothesis $[5,6]$. In addition, one needs further accurate observations to fully confirm (or disprove) the validity of general relativity in the high curvature regime such as black hole systems and other massive objects. Therefore, in recent years, more attentions have been focused on alternative theories of gravity. The most considerable alternative theories of gravity are the scalartensor theories. One of the good examples of these theories is Brans-Dicke (BD) theory which was introduced in 1961 to combine Mach's principle with Einstein's theory of gravity [7]. It is worthwhile to mention that BD theory is one of the modified theories of general relativity which can be used for several cosmological problems like inflation, cosmic acceleration, and dark energy modeling [8-10]. Also, it has a customizable parameter $(\omega)$ which indicates the strength of coupling between the matter and scalar fields. The action of 4-dimensional BD theory can be written as

$$
S=\frac{1}{16 \pi} \int d^{4} x \sqrt{-g}\left(\Phi R-\frac{\omega}{\Phi}(\nabla \Phi)^{2}\right),
$$

where $R$ and $\Phi$ are, respectively, the Ricci scalar and selfgravitating scalar field. It is interesting to note that 4dimensional stationary vacuum BD solution is just the Kerr solution with a trivial scalar field [11]. In addition, Cai and Myung proved that 4-dimensional solution of BD-Maxwell theory reduces to the Reissner-Nordström solution with a constant scalar field [12-15]. However, the solutions of BDMaxwell gravity in higher dimensions will be reduced to the Reissner-Nordström solutions with a nontrivial scalar field because of the fact that higher dimensional stress energy tensor of Maxwell field is not traceless (conformally invariant). One of the most prominent problems which makes $\mathrm{BD}$ theory nonstraightforward is the fact that the field equations of this theory are highly nonlinear. To deal with this issue, one could apply conformal transformation on known solutions of other modified theories like dilaton gravity [16]. For instance, nonlinearly charged dilatonic black hole solutions and their BD counterpart in an energy dependent space-time have been obtained by applying a conformal transformation [17].

The first attempt for modifying the Maxwell theory to a consistent theory for describing point charges was made in 
1912 by Mie $[18,19]$. After that, Born and Infeld introduced a gauge-invariant nonlinear electrodynamic model to find a classical theory of point-like charges with finite energy density [20]. Born-Infeld (BI) theory was more interesting since it was obtained by using loop correction analysis of Quantum Field Theory. Recently, Tseytlin has shown that BI theory can be derived as an effective theory of some string theory models [21-26]. Nowadays, the effects of BI electrodynamics coupled to various gravity theories have been considered by many authors in the context of black holes [27-51], rotating black branes [14, 52-57], wormholes [5861], superconductors [62-67], and other aspects of physics $[68,69]$.

On the other side, black hole thermodynamics became an interesting topic after the works of Hawking and Bekenstein [70-75]. Besides, based on the AdS/CFT correspondence, black hole thermodynamics was considered as the first step for constructing quantum gravity. In recent years, phase transition and critical behavior of the black holes have attracted more attention among physicists. Generally, at the critical point where phase transition occurs, one may find a discontinuity of state space variable such as heat capacity [76]. In addition to heat capacity, there are various approaches for studying phase transition. One of such interesting methods is based on geometrical technique. Geometrical thermodynamic method was started by Gibbs and Caratheodory [77]. Regarding this method, one could build a phase space by employing thermodynamical potential and its corresponding extensive parameter. Meanwhile, divergence points of Ricci scalar of thermodynamical metric provide information about phase transition points of thermodynamical systems.

For the first time, Weinhold introduced a new metric on the equilibrium thermodynamical phase space $[78,79]$ and after that another thermodynamical metric was defined by Ruppeiner from a different point of view [80, 81]. It is worthwhile to mention that there is a conformal relation between Ruppeiner and Weinhold metrics with the inverse of temperature as a conformal factor [82]. None of Weinhold and Ruppeiner metrics were invariant under Legendre transformation. Recently, Quevedo [83, 84] removed some problems of Weinhold and Ruppeiner methods by proposing a Legendre invariant thermodynamical metric. Although Quevedo could solve some problems which previous metrics were involved with, it has been confronted with another problems in some specific systems. To solve these problems, a new method was proposed in [85-87] which is known as HPEM metric. It was shown that HPEM metric is completely consistent with the results of the heat capacity in canonical ensemble in different gravitational systems.

In this paper, we are going to consider black hole solutions of BD-BI as well as Einstein-BI-dilaton gravity and study their phase transition based on geometrical thermodynamic methods. We compare our results with those of other methods such as extended phase space thermodynamics.

\section{Field Equation and Conformal Transformations}

The $(n+1)$-dimensional BD-BI theory action containing a scalar field $\Phi$ and a self-interacting potential $V(\Phi)$ is as follows:

$$
\begin{aligned}
& I_{\mathrm{BD}-\mathrm{BI}}=-\frac{1}{16 \pi} \int_{M} d^{n+1} x \\
& \cdot \sqrt{-g}\left(\Phi R-\frac{\omega}{\Phi}(\nabla \Phi)^{2}-V(\Phi)+\mathscr{L}(\mathscr{F})\right)
\end{aligned}
$$

where $\omega$ is a coupling constant and $\mathscr{L}(\mathscr{F})$ is the BI theory Lagrangian

$$
\mathscr{L}(\mathscr{F})=4 \beta^{2}\left(1-\sqrt{1+\frac{\mathscr{F}}{2 \beta^{2}}}\right),
$$

in which $\beta$ and $\mathscr{F}=F_{\mu \nu} F^{\mu \nu}$ are BI parameter and Maxwell invariant, respectively. It is worth mentioning that $\mathscr{L}(\mathscr{F})$ will be reduced to the standard Maxwell form $\mathscr{L}(\mathscr{F})=-\mathscr{F}$ as $\beta \rightarrow \infty$. The field equations of gravitational, scalar, and electromagnetic fields can be obtained by varying the action (2):

$$
\begin{aligned}
& G_{\mu \nu}=\frac{\omega}{\Phi^{2}}\left(\nabla_{\mu} \Phi \nabla_{\nu} \Phi-\frac{1}{2} g_{\mu \nu}(\nabla \Phi)^{2}\right)-\frac{V(\Phi)}{2 \Phi} g_{\mu \nu} \\
& +\frac{1}{\Phi}\left(\nabla_{\mu} \nabla_{\nu} \Phi-g_{\mu \nu} \nabla^{2} \Phi\right)+\frac{2}{\Phi}\left(\frac{F_{\mu \lambda} F_{\nu}{ }^{\lambda}}{\sqrt{1+\mathscr{F} / 2 \beta^{2}}}\right. \\
& \left.+\frac{1}{4} g_{\mu \nu} \mathscr{L}(\mathscr{F})\right) \\
& \nabla^{2} \Phi=\frac{1}{2[(n-1) \omega+n]}\left((n-1) \Phi \frac{d V(\Phi)}{d \Phi}\right. \\
& \left.-(n+1) V(\Phi)+(n+1) \mathscr{L}(\mathscr{F})+\frac{4 \mathscr{F}}{\sqrt{1+\mathscr{F} / 2 \beta^{2}}}\right), \\
& \nabla_{\mu}\left(\frac{F^{\mu \nu}}{\sqrt{1+\mathscr{F} / 2 \beta^{2}}}\right)=0
\end{aligned}
$$

It is not easy to solve (4)-(6) because there exists second order of scalar field in the denominator of field equation (4). In order to overcome such a problem, we can use a suitable conformal transformation and convert the BD-BI theory 
to the Einstein-BI-dilaton gravity. The suitable conformal transformation is as follows:

$$
\begin{aligned}
\bar{g}_{\mu \nu} & =\Phi^{2 /(n-1)} g_{\mu \nu}, \\
\bar{\Phi} & =\frac{n-3}{4 \alpha} \ln \Phi, \\
\alpha & =\frac{(n-3)}{\sqrt{4(n-1) \omega+4 n}} .
\end{aligned}
$$

The Einstein-BI-dilaton gravity action and its related field equations can be obtained from the BD-BI action and its related field equations by applying the mentioned conformal transformation [17]:

$$
\begin{aligned}
\bar{I}_{G} & =-\frac{1}{16 \pi} \int_{\mathscr{M}} d^{n+1} x \\
\cdot & \sqrt{-\bar{g}}\left\{\overline{\mathscr{R}}-\frac{4}{n-1}(\overline{\nabla \Phi})^{2}-\bar{V}(\bar{\Phi})+\bar{L}(\overline{\mathscr{F}}, \bar{\Phi})\right\}, \\
\overline{\mathscr{R}}_{\mu \nu} & =\frac{4}{n-1}\left(\bar{\nabla}_{\mu} \overline{\Phi \nabla_{\nu}} \bar{\Phi}+\frac{1}{4} \bar{V}(\bar{\Phi}) \bar{g}_{\mu \nu}\right)-\frac{1}{n-1} \\
& \bar{L}(\overline{\mathscr{F}}, \bar{\Phi}) \bar{g}_{\mu \nu} \\
& +\frac{2 e^{-4 \alpha \bar{\Phi} /(n-1)}}{\sqrt{1+\bar{Y}}}\left(\bar{F}_{\mu \eta} \bar{F}_{\nu}^{\eta}-\frac{\overline{\mathscr{F}}}{n-1} \bar{g}_{\mu \nu}\right), \\
\bar{\nabla}^{2} \bar{\Phi} & =\frac{n-1}{8} \frac{\partial \bar{V}(\bar{\Phi})}{\partial \bar{\Phi}} \\
& +\frac{\alpha}{2(n-3)}\left((n+1) \bar{L}(\overline{\mathscr{F}}, \bar{\Phi})+\frac{4 e^{-4 \alpha \bar{\Phi} /(n-1)} \overline{\mathscr{F}}}{\sqrt{1+\bar{Y}}}\right), \\
\bar{\nabla}_{\mu} & \left(\frac{e^{-4 \alpha \bar{\Phi} /(n-1)}}{\sqrt{1+\bar{Y}}} \bar{F}^{\mu \nu}\right)=0,
\end{aligned}
$$

where $\bar{\nabla}$ is the covariant differentiation with respect to the metric $\bar{g}_{\mu \nu}$ and $\overline{\mathscr{R}}$ is its Ricci scalar. The potential $\bar{V}(\bar{\Phi})$ and the Lagrangian $\bar{L}(\bar{F}, \bar{\Phi})$ will take the following forms [17]:

$$
\begin{gathered}
\bar{V}(\bar{\Phi})=\Phi^{-(n+1) /(n-1)} V(\Phi) \\
\bar{L}(\overline{\mathscr{F}}, \bar{\Phi})=4 \beta^{2} e^{-4 \alpha(n+1) \bar{\Phi} /[(n-1)(n-3)]}(1 \\
\left.-\sqrt{1+\frac{e^{16 \alpha \bar{\Phi} /[(n-1)(n-3)] \bar{F}}}{2 \beta^{2}}}\right) .
\end{gathered}
$$

In the limits of $\beta \rightarrow \infty$ and $\beta \rightarrow 0$, the Lagrangian will be $\bar{L}(\overline{\mathscr{F}}, \bar{\Phi})=-e^{-4 \alpha \bar{\Phi} /(n-1)} \overline{\mathscr{F}}$ and $\bar{L}(\overline{\mathscr{F}}, \bar{\Phi}) \rightarrow 0$, respectively, as expected. In previous equations, we have used the following notations:

$$
\begin{aligned}
\bar{L}(\overline{\mathscr{F}}, \bar{\Phi}) & =4 \beta^{2} e^{-4 \alpha(n+1) \bar{\Phi} /[(n-1)(n-3)]} \bar{L}(\bar{Y}), \\
\bar{L}(\bar{Y}) & =1-\sqrt{1+\bar{Y}}, \\
\bar{Y} & =\frac{e^{16 \alpha \bar{\Phi} /[(n-1)(n-3)] \overline{\mathscr{F}}}}{2 \beta^{2}} .
\end{aligned}
$$

By considering the conformal relation between these two theories, it can be understood that if $\left(\bar{g}_{\mu \nu}, \bar{F}_{\mu \nu}, \bar{\Phi}\right)$ are the solutions to the field equations of Einstein-BI-dilaton gravity (9)-(11), then the solutions of BD-BI theory could be obtained by the following form:

$$
\begin{aligned}
& {\left[g_{\mu \nu}, F_{\mu \nu}, \Phi\right]} \\
& =\left[\exp \left(-\frac{8 \alpha \bar{\Phi}}{(n-1)(n-3)}\right) \bar{g}_{\mu \nu}, \bar{F}_{\mu \nu}, \exp \left(\frac{4 \alpha \bar{\Phi}}{n-3}\right)\right] .
\end{aligned}
$$

\subsection{Black Hole Solutions in Einstein-BI-Dilaton Gravity and BD-BI Theory}

2.1.1. Einstein Frame. In this section, we briefly obtain the Einstein-BI-dilaton gravity solutions and then by using the conformal transformation, we calculate the solutions of $\mathrm{BD}$ BI theory [88]. We assume the following metric with various horizon topology:

$$
d \bar{s}^{2}=-Z(r) d t^{2}+\frac{d r^{2}}{Z(r)}+r^{2} R^{2}(r) d \Omega_{k}^{2},
$$

where $d \Omega_{k}^{2}$ is an $(n-1)$-dimensional hypersurface of Euclidean metric with constant curvature $(n-1)(n-2) k$ and volume $\omega_{n-1}$ with the following explicit form:

$$
\begin{aligned}
& d \Omega_{k}^{2} \\
& = \begin{cases}d \theta_{1}^{2}+\sum_{i=2}^{n-1} \prod_{j=1}^{i-1} \sin ^{2} \theta_{j} d \theta_{i}^{2} & k=1 \\
d \theta_{1}^{2}+\sinh ^{2} \theta_{1} d \theta_{2}^{2}+\sinh ^{2} \theta_{1} \sum_{i=3}^{n-1} \prod_{j=2}^{i-1} \sin ^{2} \theta_{j} d \theta_{i}^{2} & k=-1 \\
\sum_{i=1}^{n-1} d \phi_{i}^{2} & k=0 .\end{cases}
\end{aligned}
$$

In order to obtain consistent solutions, we should consider a suitable functional form for the potential, $\overline{\mathbf{V}}(\bar{\Phi})$. It was shown that the proper potential is a Liouville-type one with both topological and BI correction terms, as [17]

$$
\begin{aligned}
\overline{\mathbf{V}}(\bar{\Phi})= & 2 \Lambda \exp \left(\frac{4 \alpha \bar{\Phi}}{n-1}\right) \\
& +\frac{k(n-1)(n-2) \alpha^{2}}{b^{2}\left(\alpha^{2}-1\right)} \exp \left(\frac{4 \bar{\Phi}}{(n-1) \alpha}\right) \\
& +\frac{W(r)}{\beta^{2}} .
\end{aligned}
$$


It is notable to mention that, in the limit of $\alpha \rightarrow 0$ (absence of dilaton field) and $\beta \rightarrow \infty, \overline{\mathbf{V}}(\bar{\Phi})$ reduces to $2 \Lambda$, as expected [13]. Now, regarding the field equations (9)-(11), metric (16), and the potential $\overline{\mathbf{V}}(\bar{\Phi})$, it is a matter of calculation to show that

$$
\begin{aligned}
& F_{t r}=E(r) \\
& =\frac{q e^{(4 \alpha \bar{\Phi}(r) /(n-1))}}{[r R(r)]^{(n-1)} \sqrt{1+e^{(8 \alpha \bar{\Phi}(r) /(n-3))} q^{2}[r R(r)]^{-2(n-1)} / \beta^{2}}}, \\
& \bar{\Phi}=\frac{(n-1) \alpha}{2\left(1+\alpha^{2}\right)} \ln \left(\frac{b}{r}\right), \\
& W(r)=\frac{4 q(n-1) \beta^{2} R(r)}{\left(1+\alpha^{2}\right) r^{\gamma} b^{n \gamma}} \int \frac{E(r)}{r^{n(1-\gamma)-\gamma}} d r \\
& +\frac{4 \beta^{4}}{R(r)^{2(n+1) /(n-3)}}\left(1-\frac{E(r) R(r)^{(n-3)}}{q r^{1-n}}\right) \\
& -\frac{4 q \beta^{2} E(r)}{r^{n-1}}\left(\frac{r}{b}\right)^{\gamma(n-1)}, \\
& Z(r)=-\frac{k(n-2)\left(\alpha^{2}+1\right)^{2}(r / b)^{2 \gamma}}{\left(\alpha^{2}+n-2\right)\left(\alpha^{2}-1\right)}+\left(\frac{\left(1+\alpha^{2}\right)^{2} r^{2}}{(n-1)}\right) \\
& \cdot \frac{2 \Lambda(r / b)^{-2 \gamma}}{\left(\alpha^{2}-n\right)}-\frac{m}{r^{(n-1)(1-\gamma)-1}} \\
& -\frac{4\left(1+\alpha^{2}\right)^{2} q^{2}(r / b)^{2 \gamma(n-2)}}{\left(n-\alpha^{2}\right) r^{2(n-2)}}\left(\frac{1}{2(n-1)} F_{1}(\eta)\right. \\
& \left.-\frac{1}{\alpha^{2}+n-2} F_{2}(\eta)\right), \\
& R(r)=\exp \left(\frac{2 \alpha \bar{\Phi}}{n-1}\right)=\left(\frac{b}{r}\right)^{\gamma},
\end{aligned}
$$

where $m$ is an integration constant related to mass and $b$ is another constant related to scalar field, and

$$
\begin{aligned}
& F_{1}(\eta) \\
& \quad={ }_{2} F_{1}\left(\left[\frac{1}{2}, \frac{(n-3) \Upsilon}{\alpha^{2}+n-2}\right],\left[1+\frac{(n-3) \Upsilon}{\alpha^{2}+n-2}\right],-\eta\right), \\
& \quad={ }_{2} F_{1}\left(\left[\frac{1}{2}, \frac{(n-3) \Upsilon}{2(n-1)}\right],\left[1+\frac{(n-3) \Upsilon}{2(n-1)}\right],-\eta\right), \\
& \Upsilon= \\
& \eta=\frac{\alpha^{2}+n-2}{2 \alpha^{2}+n-3}, \\
& \gamma=\frac{q^{2}(r / b)^{2 \gamma(n-1)(n-5) /(n-3)}}{\beta^{2} r^{2(n-1)}}
\end{aligned}
$$

It is worthwhile to mention that the dilatonic Maxwell solutions [89] can be achieved from the obtained solutions in the limit of $\beta \rightarrow \infty$. The divergencies of scalar curvatures at the origin guarantee the existence of singularity. We interpret such a singularity as black hole since it is covered by an event horizon [17].

2.1.2. Jordan Frame. To obtain the black hole solutions of BDBI theory, first, by using the conformal transformation (12), $\mathbf{V}(\Phi)$ would be

$$
\begin{aligned}
\mathbf{V}(\Phi)= & 2 \Lambda \Phi^{2} \\
& +\frac{k(n-1)(n-2) \alpha^{2}}{b^{2}\left(\alpha^{2}-1\right)} \Phi^{\left[(n+1)\left(1+\alpha^{2}\right)-4\right] /\left[(n-1) \alpha^{2}\right]} \\
& +\Phi^{(n+1) /(n-1)} \frac{W(r)}{\beta^{2}}
\end{aligned}
$$

Also, by considering the following $(n+1)$-dimensional metric,

$$
d s^{2}=-A(r) d t^{2}+\frac{d r^{2}}{B(r)}+r^{2} H^{2}(r) d \Omega_{k}^{2}
$$

one can find the following solutions through conformal transformation:

$$
\begin{aligned}
& A(r)=\left(\frac{r}{b}\right)^{4 \gamma /(n-3)} Z(r), \\
& B(r)=\left(\frac{r}{b}\right)^{-4 \gamma /(n-3)} Z(r), \\
& H(r)=\left(\frac{r}{b}\right)^{-\gamma((n-5) /(n-3))}, \\
& \Phi(r)=\left(\frac{r}{b}\right)^{-2 \gamma(n-1) /(n-3)} .
\end{aligned}
$$

It is notable that, like Einstein frame, these solutions can be interpreted as black holes which are covered by event horizon.

\section{Thermodynamic Properties: Dilatonic-BI versus BD-BI Black Holes}

3.1. Thermodynamic Quantities. In the following, we give a brief review regarding thermodynamic quantities of the black hole solutions in both frames. The Hawking temperature of the black hole can be obtained by using the surface gravity interpretation $(\kappa)$ through the following relation:

$$
\begin{aligned}
T & =\frac{\kappa}{2 \pi}=\frac{1}{2 \pi} \sqrt{-\frac{1}{2}\left(\nabla_{\mu} \chi_{\nu}\right)\left(\nabla^{\mu} \chi^{\nu}\right)} \\
& = \begin{cases}\frac{Z^{\prime}\left(r_{+}\right)}{4 \pi}, & \text { dilatonic BI } \\
\frac{1}{4 \pi} \sqrt{\frac{B(r)}{A(r)}} A^{\prime}\left(r_{+}\right), & \text {BD-BI, }\end{cases}
\end{aligned}
$$


in which $\chi=\partial / \partial t$ is the time like null Killing vector. It is easy to show that the Hawking temperature in both frames is uniform as

$$
\begin{aligned}
T & =\frac{\left(\alpha^{2}+1\right)}{2 \pi(n-1)}\left[\frac{-k(n-2)(n-1)}{2\left(\alpha^{2}-1\right) r_{+}}\left(\frac{b}{r_{+}}\right)^{-2 \gamma}\right. \\
& \left.-\Lambda r_{+}\left(\frac{b}{r_{+}}\right)^{2 \gamma}+\Gamma_{+}\right], \text {dilatonic BI \& BD-BI, }
\end{aligned}
$$

where

$$
\Gamma_{+}=-\frac{\left(\alpha^{2}+1\right)^{2} q^{2}}{2 \pi(n-1)}\left(\frac{r_{+}}{b}\right)^{2 \gamma(n-2)} r_{+}^{3-2 n} F_{1}\left(\eta_{+}\right) .
$$

Following $[12,17]$, the finite mass and entropy of the black hole in both Einstein and Jordan frames are

$$
\begin{aligned}
M & =\frac{\omega_{n-1} b^{(n-1) \gamma}}{16 \pi}\left(\frac{n-1}{1+\alpha^{2}}\right) m, \\
S & =\frac{\omega_{n-1} b^{(n-1) \gamma}}{4} r_{+}^{(n-1)(1-\gamma)} .
\end{aligned}
$$

In addition, the electric charge $Q$ of the black holes can be obtained via Gauss's law

$$
Q=\frac{q}{4 \pi}
$$

Also, one can obtain the electric potential as

$$
\begin{gathered}
U=\left(\frac{r_{+}}{b}\right)^{4 \gamma+1} \frac{b \beta\left(\alpha^{2}+1\right)}{\left(5 \alpha^{2}+1\right)}{ }_{2} F_{1}\left(\left[\frac{1}{2}, \frac{5 \alpha^{2}+1}{6\left(2 \alpha^{2}+1\right)}\right],\right. \\
\left.\left[\frac{17 \alpha^{2}+7}{6\left(2 \alpha^{2}+1\right)}\right], \frac{\beta^{2} b^{6}}{q^{2}}\left(\frac{r_{+}}{b}\right)^{6 \gamma+6}\right) .
\end{gathered}
$$

It is straightforward to show that the mentioned conserved and thermodynamical quantities satisfy the first law of thermodynamics as

$$
d M=T d S+U d Q
$$

3.2. Heat Capacity and Thermal Stability. Here, we want to investigate thermal stability of the black holes. Due to the set of state functions and thermodynamic variables of a system, one may study the thermodynamic stability from different points of view through various ensembles. One of the common methods to study phase transition is regarding the canonical ensemble. In this ensemble, thermal stability of a system will be ensured by positivity of the heat capacity. One can obtain the heat capacity relation with fixed charge as

$$
C_{\mathrm{Q}}=\frac{(\partial M / \partial S)_{\mathrm{Q}}}{\left(\partial^{2} M / \partial S^{2}\right)_{\mathrm{Q}}}=\frac{M_{S}}{M_{S S}}=T\left(\frac{\partial S}{\partial T}\right)_{\mathrm{Q}},
$$

where $M_{S}=\partial M / \partial S$ and $M_{S S}=\partial^{2} M / \partial S^{2}$.

From the nominator of heat capacity, it is evident that the temperature $\left(M_{S}\right)$ has crucial role on the sign of $C_{Q}$. In addition, divergence points of heat capacity are indicating second-order phase transition. Hence, these divergencies are utilized for calculating critical values and investigating the critical behavior of the black hole. Now, for studying phase transition, we introduce various geometrical thermodynamic methods and compare their results with those arisen from the heat capacity.

3.3. Geometrical Study of the Phase Transition. One of the basic motivations for considering the geometrical thermodynamics comes from the fact that this formalism helps us to describe in an invariant way the thermodynamic properties of a given thermodynamical system in terms of geometric structures. Also, this method is a strong machinery for describing phase transition of the black holes. Another motivation is to give an independent picture regarding thermodynamical aspects of a system. In addition to some useful information about bound points, phase transitions, and thermal stability conditions, this method contains information regarding molecular interaction around phase transitions for thermodynamical systems. In other words, by studying the sign of thermodynamical Ricci scalar around phase transition points, one can extract information whether interaction is repulsive or attractive. Based on such motivations, it will be interesting to investigate black hole phase transition in the context of geometrical thermodynamics, as an independent approach.

In order to study the phase transition, one can employ thermodynamical quantities to build geometrical space-time. There are several metrics in the context of geometrical thermodynamics which one can use to study phase transition and critical behavior. The well-known thermodynamical metrics are Weinhold, Ruppeiner, Quevedo, and HPEM as the recently proposed method. As we mentioned, in some specific types of systems, the Weinhold, Ruppeiner, and Quevedo metrics are not applicable and they will face some problems. Here, we want to discuss these thermodynamical metrics and their possible mismatched problems.

Thermodynamical metric was first introduced by Weinhold $[78,79]$. This thermodynamical metric is given by

$$
d S_{W}^{2}=g_{a b}^{W} d X^{a} d X^{b}
$$

where $g_{a b}^{W}=\partial^{2} M\left(X^{c}\right) / \partial X^{a} \partial X^{b}, X^{a} \equiv X^{a}\left(S, N^{i}\right)$, and $N^{i}$ denotes other extensive variables of the system. By calculating $M$ as a function of extensive quantities (such as entropy and electric charge) and using Weinhold metric (32), one can find the Ricci scalar. It is expected that the singular points of the Weinhold Ricci scalar match the root or divergence points of the heat capacity, to indicate the bound point or the phase transition ones. We plot Figure 1 to investigate the mentioned behavior.

After that, Ruppeiner [80, 81] has defined another thermodynamical metric with the following form:

$$
d S_{R}^{2}=g_{a b}^{R} d X^{a} d X^{b}
$$

where $g_{a b}^{R}=-\partial^{2} S\left(X^{c}\right) / \partial X^{a} \partial X^{b}$ and $X^{a} \equiv X^{a}\left(M, N^{i}\right)$. 

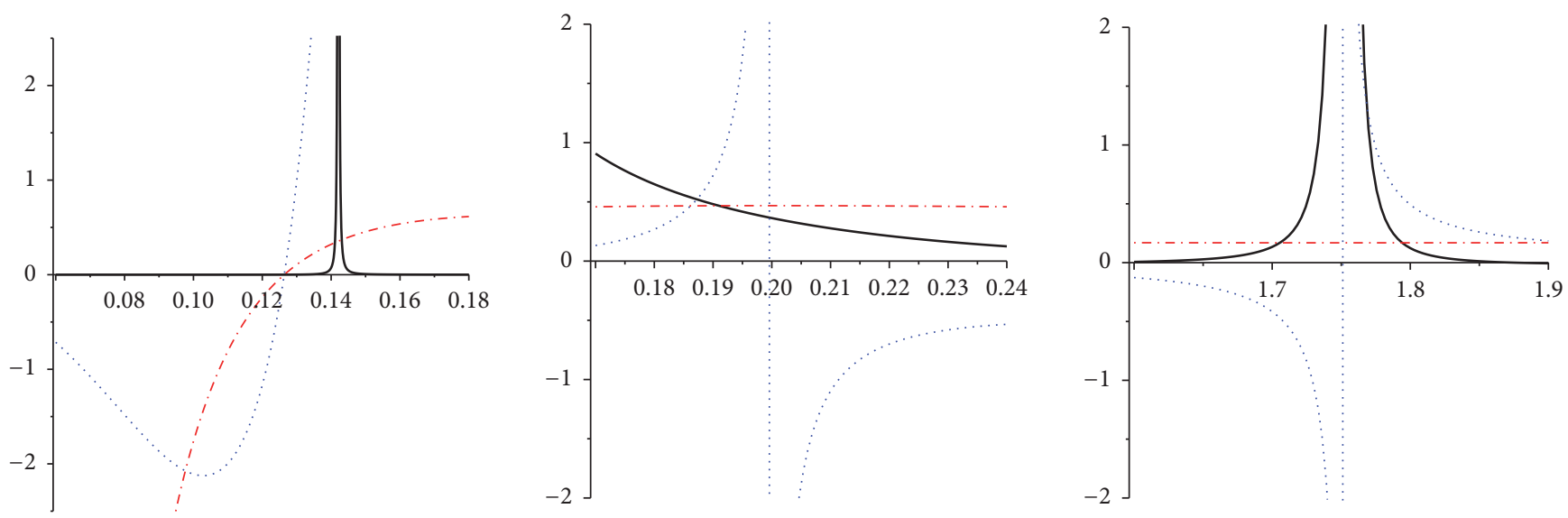

Figure 1: Weinhold metric. $\mathscr{R}$ (continuous line), $C_{Q}$ (dotted line), and $T$ (dot dashed) versus $r_{+}$for $\Lambda=-1, n=4, q=0.1, b=1, \omega=10$, and $\beta=1.5$. "Note: all three panels are plotted with the same parameters, but different regions and scales."
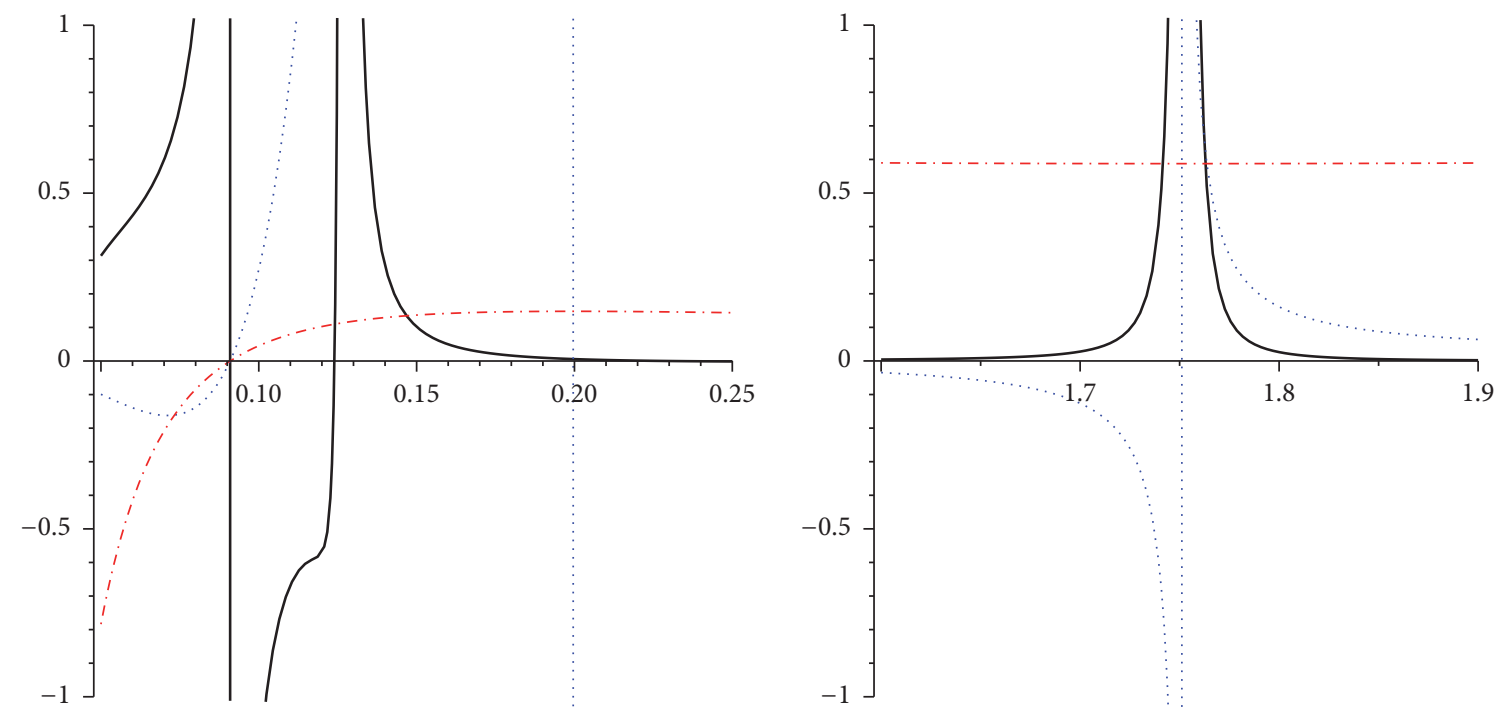

Figure 2: Ruppeiner metric. $\mathscr{R}$ (continuous line), $C_{\mathrm{Q}}$ (dotted line), and $T$ (dot dashed) versus $r_{+}$for $\Lambda=-1, n=4, q=0.1, b=1, \omega=10$, and $\beta=1.5$. "Note: both panels are plotted with the same parameters, but different regions and scales."

In the Ruppeiner metric, thermodynamical potential is entropy. It is worthwhile mentioning that these two metrics are conformally related to each other [82]. We plot Figure 2 to show that the Ruppeiner Ricci scalar divergencies are not matched with those of heat capacity.

As we have shown, calculating thermodynamical Ricci scalar of these two thermodynamical metrics indicates that the results were not completely consistent with the results of heat capacity in the canonical ensemble. In order to remove some failures of the Weinhold and Ruppeiner metrics, recently, another metric which is Legendre invariant has been introduced by Quevedo $[83,84]$. The Quevedo metric has the following form:

$$
d s_{\mathrm{Q}}^{2}=\Omega\left(-M_{S S} d S^{2}+M_{\mathrm{QQ}} d \mathrm{Q}^{2}\right),
$$

where the conformal coefficient $\Omega$ is

$$
\Omega=\left(S M_{S}+Q M_{Q}\right) .
$$

Considering Figures 1-3, we find that, by using these three well-known metrics, there is at least a mismatch between heat capacity divergencies and thermodynamical Ricci scalar divergencies (of these three metrics). Therefore, these metrics are not appropriate tools for investigation of our black hole phase transitions and related critical behavior. In other words, the method of geometrical thermodynamics which has been reported in [90] is not an applicable method in the scalar field theory.

Very recently, a new metric was proposed by Hendi et al. (HPEM metric) to solve this problem. This method is applied for various gravitating systems and it is shown that the root and divergence points of the heat capacity coincide with the divergence points of the HPEM Ricci scalar (see 

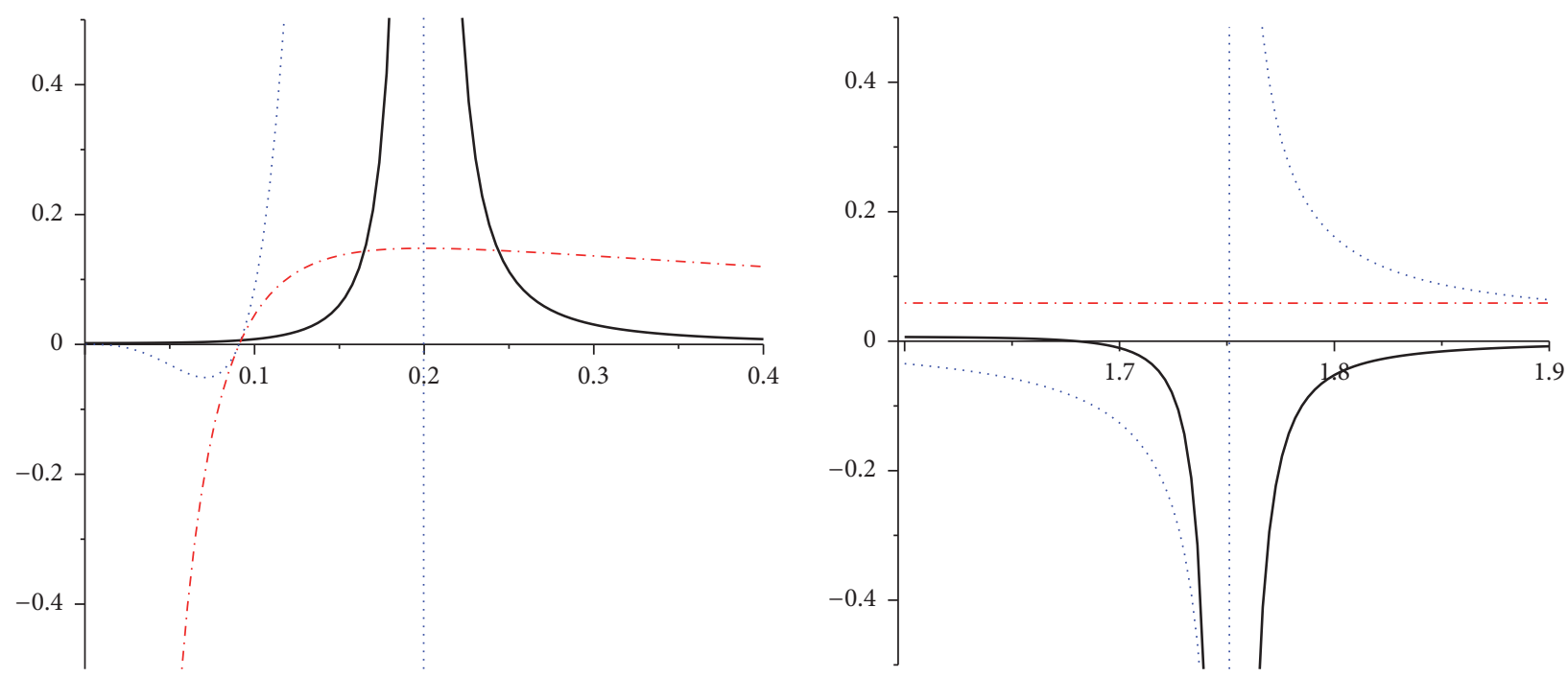

FIGURE 3: Quevedo metric. $\mathscr{R}$ (continuous line), $C_{Q}$ (dotted line), and $T$ (dot dashed) versus $r_{+}$for $\Lambda=-1, n=4, q=0.1, b=1, \omega=10$, and $\beta=1.5$. "Note: both panels are plotted with the same parameters, but different regions."

Figures 4-6, for more details). The generalized HPEM metric with $n$ extensive variables $(n \geq 2)$ has the following form [8587]:

$$
\begin{aligned}
& d s_{\mathrm{HPEM}}^{2}=\frac{S M_{S}}{\left(\prod_{i=2}^{n}\left(\partial^{2} M / \partial \chi_{i}^{2}\right)\right)^{3}}\left(-M_{S S} d S^{2}\right. \\
& \left.\quad+\sum_{i=2}^{n}\left(\frac{\partial^{2} M}{\partial \chi_{i}^{2}}\right) d \chi_{i}^{2}\right)
\end{aligned}
$$

where $\chi_{i}^{\prime}$ s $\left(\chi_{i} \neq S\right)$ are extensive parameters. It is notable that HPEM metric is the same as that presented by Quevedo (with the same ",,,$-++ \ldots$ " signature), but with different conformal factor, and therefore it is expected to enjoy Legendre invariance. In what follows, we will investigate the stability and phase transition of the physical BD-BI black holes in the context of the heat capacity and geometrical thermodynamics by using HPEM metric.

As the first significant point which must be taken into deep consideration, one should regard the sign of the temperature. The positivity of the temperature denotes a physical black hole, whereas the negativity of $T$ represents a nonphysical system. The temperature behavior has been shown in the figures, too. As we can see, there is a lower bound for the horizon radius $\left(r_{0}\right)$, in which for $r_{+}<r_{0}$, we encounter a nonphysical black hole, owing to negative sign of temperature. In contrast, in the case of $r_{+}>r_{0}$, we confront a physical system due to the positivity of the temperature. In other words, the horizon radius of physical black holes is located in this region.

Figure 4 shows that, for the special values of the electric charge, nonlinearity parameter, and BD-coupling coefficient, we can obtain three characteristic points. One of them refers to the root of heat capacity (or temperature) which is known as $r_{0}$ and others are related to the divergence points of heat capacity which are denoted as $r_{d_{1}}$ and $r_{d_{2}}$ $\left(r_{d_{1}}<r_{d_{2}}\right)$. We also find that all divergence points of the
TABLE 1: Critical points of BD-BI theory for $q=0.1, \Lambda=-1, \omega=10$, $b=1$, and $\beta=1.5$.

\begin{tabular}{cccc}
\hline$n$ & $r_{0}$ & $r_{d_{1}}$ & $r_{d_{2}}$ \\
\hline 5 & 0.2052 & 0.3820 & 2.5814 \\
6 & 0.2842 & 0.4772 & 3.5605 \\
7 & 0.3422 & 0.5390 & 4.7619 \\
\hline
\end{tabular}

Ricci scalar (for HPEM metric) coincide with these three points. Here, we use some tables to study the influences of different parameters (dimensions, nonlinearity parameter, and BD-coupling coefficient) on the mentioned characteristic points.

These tables provide information regarding the lower bound of horizon radius, two points of phase transition (for the case of $\mathrm{BD}-\mathrm{BI}$ ), and their dependencies to the variation of dimensions, nonlinearity parameter, and coupling coefficient. Regarding the tables and Figures $4-7$, it is evident that one root and two divergence points for the heat capacity are almost observed. It is worthwhile to mention that the region of $r_{0}<r_{+}<r_{d_{1}}$ (positive sign of heat capacity) shows the stability of the system. In contrast, one can find that, for the region of $r_{d_{1}}<r_{+}<r_{d_{2}}$, the heat capacity has negative sign which indicates instability. In addition, at region $r_{+}>r_{d_{2}}$, the system is in the stable state due to the positive sign of heat capacity (see Figures 4-6 for more details). According to Table 1, one can conclude that the lower bound radius and two divergence points are increasing functions of the dimensions. Also, according to Table 2, the lower bound of horizon radius and the first divergence point $\left(r_{d_{1}}\right)$ will increase by increasing $\beta$ (the nonlinearity parameter), whereas the second point of divergency remains steady over this change. Considering figures and Table 2 , it is obvious that, by increasing $\beta$, root and the first divergence point of heat capacity will increase up to a point and then any increment in this parameter would have negligible effect on these values. 

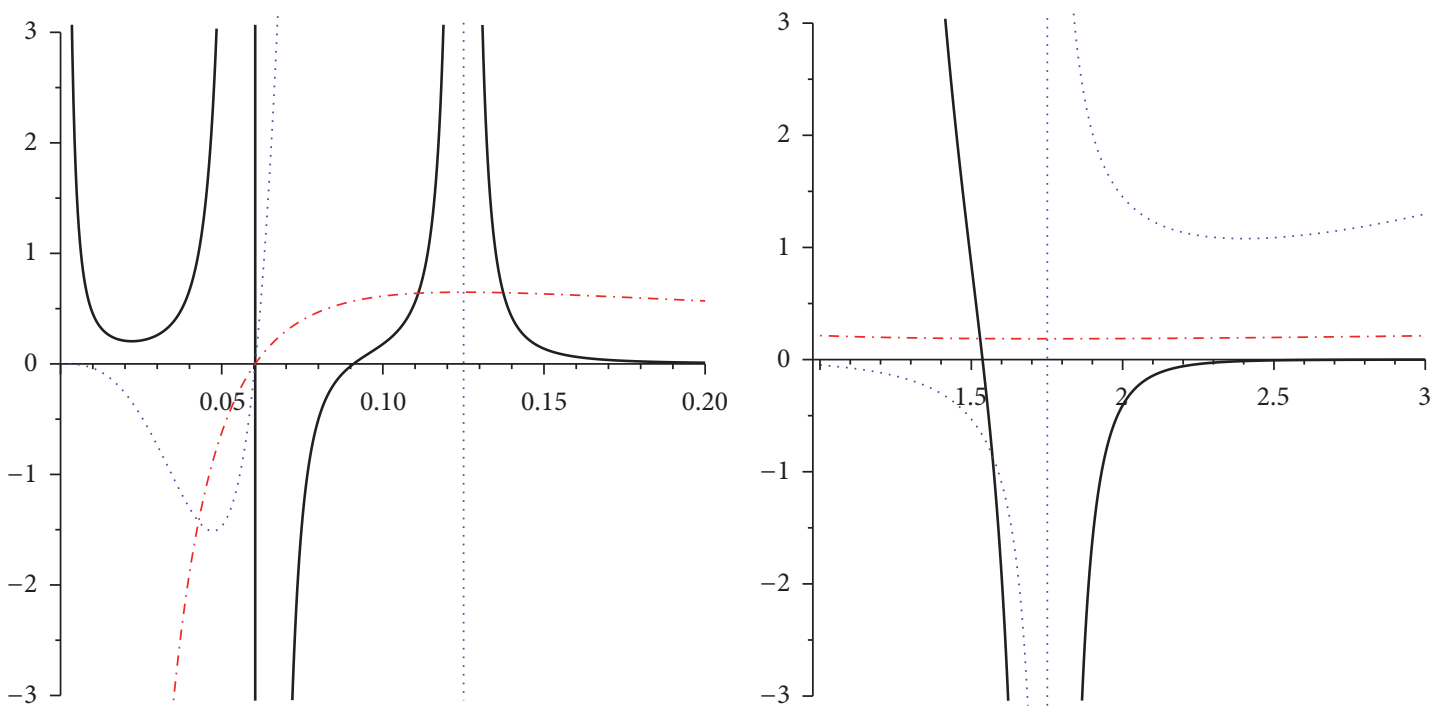

(a)
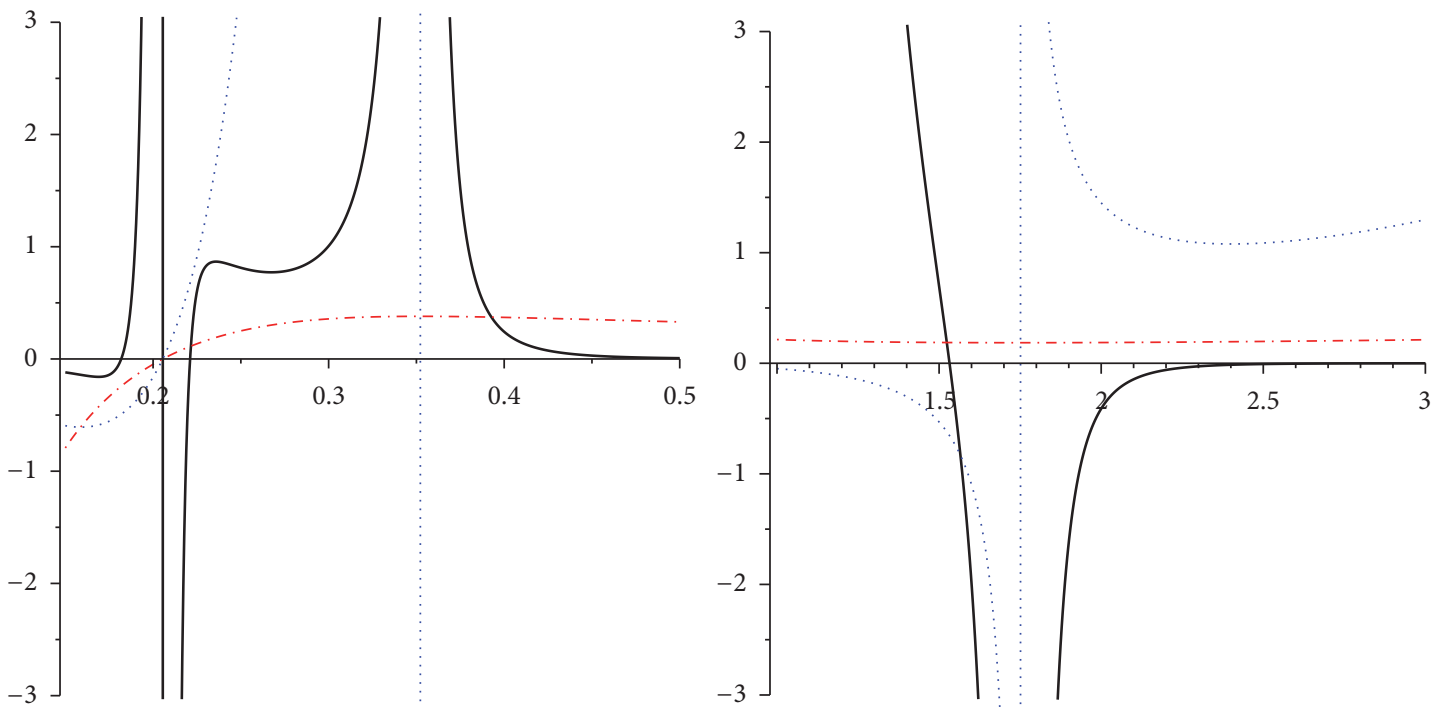

(b)

Figure 4: HPEM metric. $\mathscr{R}$ (continuous line), $C_{Q}$ (dotted line), and $T$ (dot dashed) versus $r_{+}$for $\Lambda=-1, n=4, q=0.1, b=1$, and $\omega=10$. $\beta=1$ (a) and $\beta=5$ (b). "Note: both panels in the same line are plotted with the same parameters, but different regions and scales."

To put in other words, it can be interpreted that, in large $\beta$, we will face the Brans-Dicke-Maxwell behavior [91]. For large $\beta$, the obtained values for lower bound horizon radius and divergence point are the same as the obtained values for the Brans-Dicke-Maxwell case [91]. It is notable that the unstable region (between two divergencies, where the heat capacity is negative) is larger in small $\beta$ than the large one (Brans-Dicke-Maxwell case) as it would be expected, which is due to the nature of nonlinearity that would cause the instability of system to increase. Meanwhile, $r_{0}$ and $r_{d_{1}}$ have ascending functions and $r_{d_{2}}$ will be declined by increasing $\omega$ (see Table 3). Generally, from what has been discussed above, dimensionality $n$ and BD-coupling coefficient $\omega$ are playing the main role in changes of the location of larger divergence point.
TABLE 2: Critical points of BD-BI theory for $q=0.1, \Lambda=-1, \omega=10$, $b=1$, and $n=4$.

\begin{tabular}{lccc}
\hline$\beta$ & $r_{0}$ & $r_{d_{1}}$ & $r_{d_{2}}$ \\
\hline 0.1 & 0.0056 & 0.0112 & 1.7512 \\
1.0 & 0.0604 & 0.1251 & 1.7512 \\
1.5 & 0.0902 & 0.1996 & 1.7512 \\
5.0 & 0.2056 & 0.3523 & 1.7512 \\
100.0 & 0.2400 & 0.3600 & 1.7512 \\
200.0 & 0.2400 & 0.3600 & 1.7512 \\
\hline
\end{tabular}

\section{Conclusion}

In this paper, the main goal was studying thermodynamical behavior of the BD-BI and Einstein-BI-dilaton black hole 

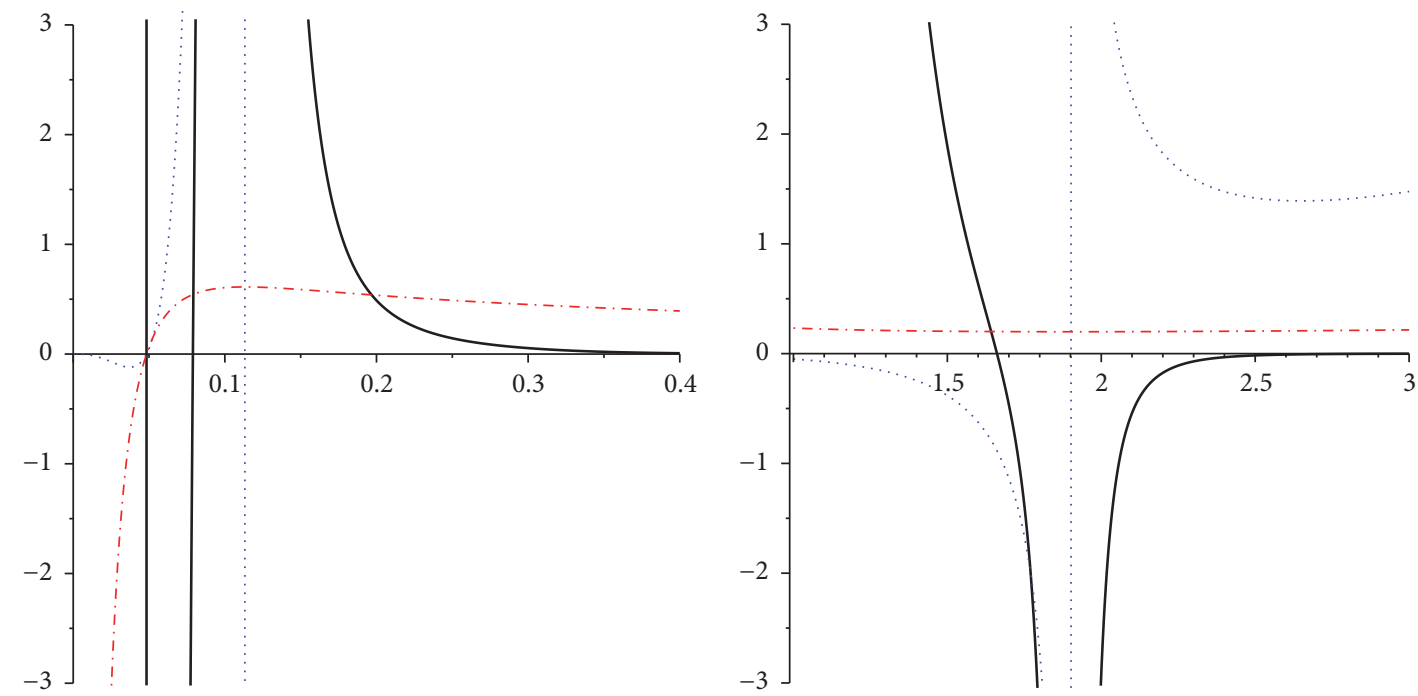

(a)
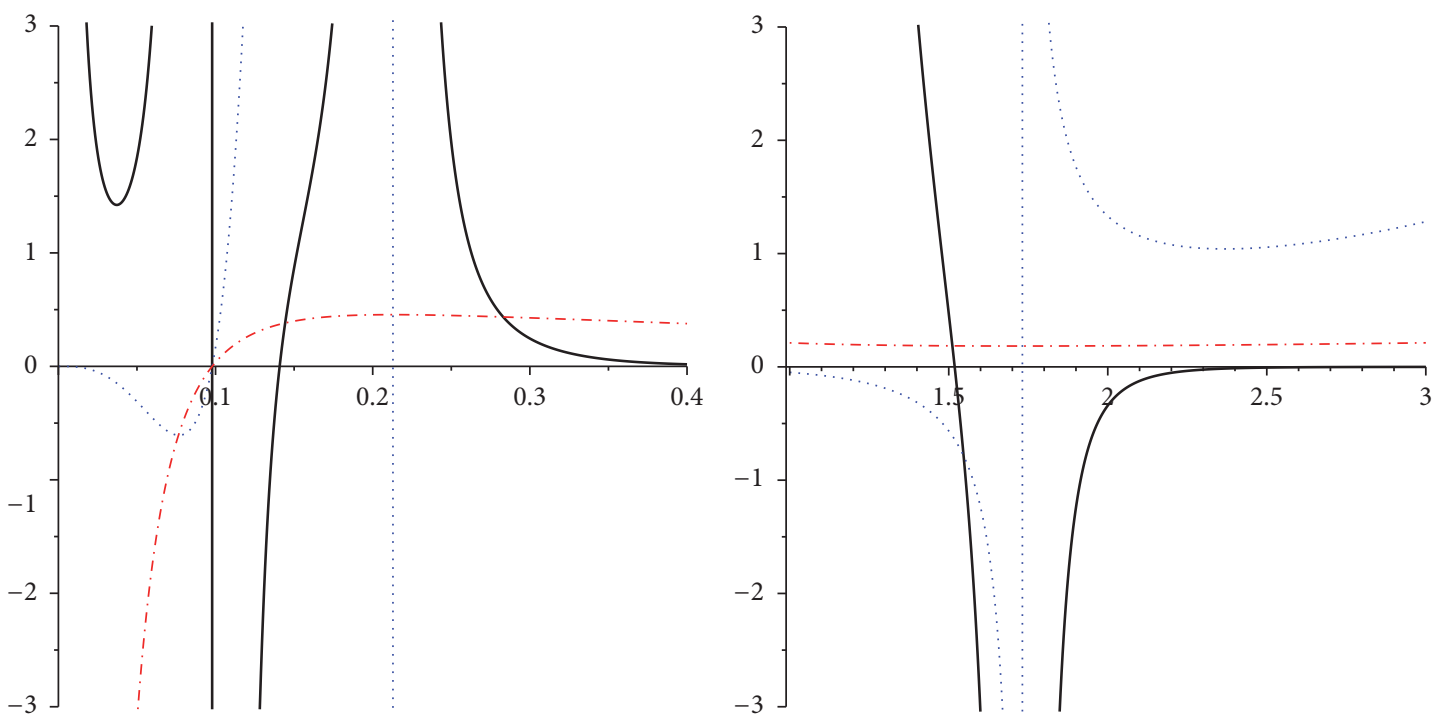

(b)

Figure 5: HPEM metric. $\mathscr{R}$ (continuous line), $C_{Q}$ (dotted line), and $T$ (dot dashed) versus $r_{+}$for $\Lambda=-1, n=4, q=0.1, b=1$, and $\beta=1.5$. $\omega=0.2$ (a) and $\omega=200$ (b). "Note: both panels in the same line are plotted with the same parameters, but different regions."

TABLE 3: Critical points of BD-BI theory for $q=0.1, \Lambda=-1, n=4$, $b=1$, and $\beta=1.5$.

\begin{tabular}{lccc}
\hline$\omega$ & $r_{0}$ & $r_{d_{1}}$ & $r_{d_{2}}$ \\
\hline 0.2 & 0.0482 & 0.1132 & 1.9022 \\
2 & 0.0742 & 0.1658 & 1.8038 \\
200 & 0.0974 & 0.2128 & 1.7318 \\
\hline
\end{tabular}

solutions. Since both of these solutions had very similar thermodynamical behavior in the context of geometrical thermodynamics, we have just considered the BD-BI ones. We have investigated the stability and phase transition in the canonical ensemble through the use of heat capacity. We have found that, for having a physical black hole (positive temperature), there should be a restriction on the value of the horizon radius, which leads to a physical limitation point. This point was a border between nonphysical and physical black hole horizon radius. Moreover, investigating the phase transition of the black holes exhibited that there exist secondorder phase transition points. In other words, the heat capacity had one real positive root and two divergence points. It was shown that these points (the root and divergence points of heat capacity) were affected by variation of the BI parameter, BD-coupling constant, and dimensions. From the presented tables and figures, we have found that the effect of dimensions on the larger divergence point was more than other factors and in contrast, the BI parameter had no sensible effect on this value. The effect of BD-coupling constant on these three points was so small in a way that, by applying a dramatic change in this constant, we observed a small change in the value of such characteristic points. 

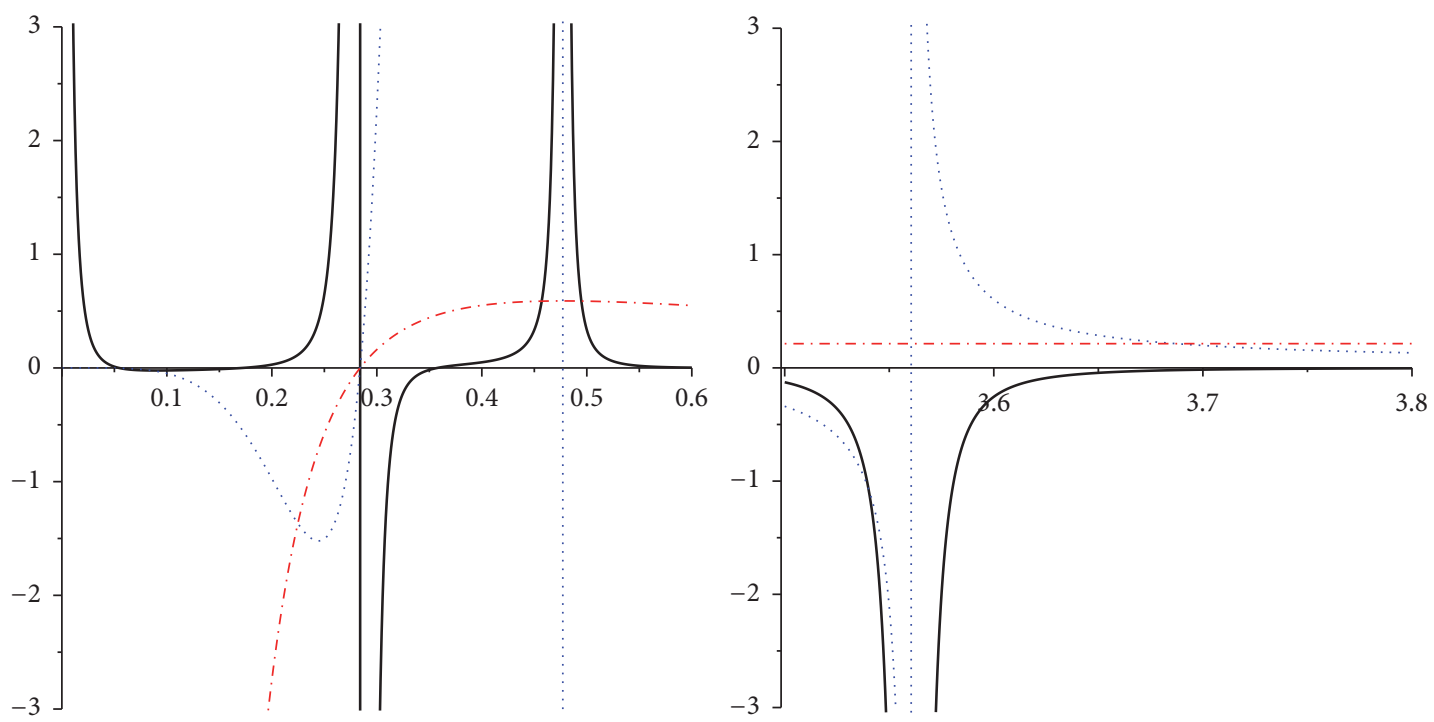

Figure 6: HPEM metric. $\mathscr{R}$ (continuous line), $C_{Q}$ (dotted line), and $T$ (dot dashed) versus $r_{+}$for $\Lambda=-1, n=6, q=0.1, b=1, \omega=10$, and $\beta=1.5$. "Note: both panels are plotted with the same parameters, but different regions and scales."
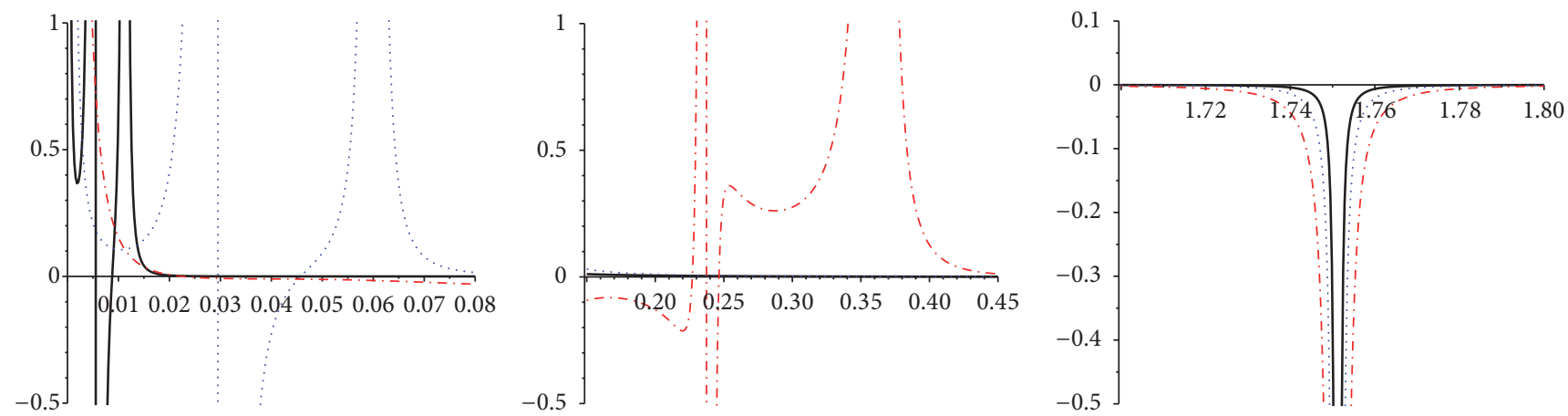

Figure 7: HPEM metric. $\mathscr{R}$ versus $r_{+}$for $\Lambda=-1, n=4, q=0.1, b=1, \omega=10$, and $\beta=0.1$ (continuous line), $\beta=0.5$ (dotted line), and Maxwell (dot dashed). "Note: all three panels are plotted with the same parameters, but different regions and scales."

It was illustrated that in the context of thermal stability there exist four regions, specified by the root and two divergence points of the heat capacity. The root of heat capacity was referred to as the lower bound of horizon radius that separated the nonphysical black holes from the physical ones. Between the two divergencies, we encountered an unstable state and after the second divergence point black hole obtained a stable state. It is notable that, for small $\beta$, because of the nonlinearity effect, the unstable region is larger than the Maxwell case (large $\beta$ ) [91].

Eventually, we employed the geometrical thermodynamic method to study the phase transition. We have shown that Weinhold, Ruppeiner, and Quevedo metrics failed to provide a consistent result with the heat capacity's result. In other words, their thermodynamical Ricci scalar's divergencies did not match the root and divergencies of the heat capacity, exactly. In some of these methods, we encountered extra divergency which did not coincide with any of the phase transition points.

At last, using the HPEM metric, we achieved desirable results. It was shown that all the divergence points of the
Ricci scalar of the mentioned metric covered the divergencies and root of the heat capacity. It is worth mentioning that the behavior of the curvature scalar was different near its divergence points. In other words, the divergence points of the Ricci scalar related to root of the heat capacity could be distinguished from the divergencies related to phase transition points based on the curvature scalar behavior.

Regarding the used method of this paper, it is interesting to extend obtained results to an energy dependent space-time and discuss the role of gravity's rainbow [92-95]. We leave this issue for future work.

\section{Conflicts of Interest}

The authors declare that there are no conflicts of interest regarding the publication of this paper.

\section{Acknowledgments}

The authors acknowledge M. Momennia and S. Panahiyan for reading the manuscript. They wish to thank Shiraz University 
Research Council. This work has been supported financially by the Research Institute for Astronomy and Astrophysics of Maragha (RIAAM), Iran.

\section{References}

[1] M. Laue, "Die nordströmsche gravitationstheorie," Jahrbuch der Radioaktivität und Elektronik, vol. 14, pp. 263-313, 1913.

[2] S. Perlmutter, G. Aldering, G. Goldhaber et al., "Measurements of $\Omega$ and $\Lambda$ from 42 high-redshift supernovae," Astrophysical Journal, vol. 517, article 565, 1999.

[3] S. Perlmutter, M. S. Turner, and M. White, "Constraining dark energy with type Ia supernovae and large-scale structure," Physical Review Letters, vol. 83, no. 4, pp. 670-673, 1999.

[4] A. G. Riess, L.-G. Strolger, J. Tonry et al., "Type ia supernova discoveries at $z>1$ from the hubble space telescope: evidence for past deceleration and constraints on dark energy evolution," Astrophysical Journal, vol. 607, no. 2, pp. 665-687, 2004.

[5] P. A. M. Dirac, "A new basis for cosmology," Proceedings of the Royal Society of London A, vol. 165, no. 921, pp. 199-208, 1938.

[6] S. Weinberg, Gravitation and Cosmology, Wiley, New York, NY, USA, 1972.

[7] C. Brans and R. H. Dicke, "Mach's principle and a relativistic theory of gravitation," Physical Review, vol. 124, no. 3, pp. 925935, 1961.

[8] N. Banerjee and D. Pavon, "Cosmic acceleration without quintessence," Physical Review D, vol. 63, no. 4, Article ID 043504, 2001.

[9] M. Sharif and S. Waheed, "Anisotropic universe models in Brans-Dicke theory," European Physical Journal C, vol. 72, no. 2, article 1876, pp. 1-12, 2012.

[10] M. K. Mak and T. Harko, "Brans-Dicke cosmology with a scalar field potential," Europhysics Letters, vol. 60, no. 1, pp. 155-161, 2002.

[11] S. W. Hawking, "Black holes in the Brans-Dicke theory of gravitation," Communications in Mathematical Physics, vol. 25, pp. 167-171, 1972.

[12] R.-G. Cai and Y. S. Myung, "Black holes in the Brans-DickeMaxwell theory," Physical Review. D. Third Series, vol. 56, no. 6, pp. 3466-3470, 1997.

[13] M. H. Dehghani, J. Pakravan, and S. H. Hendi, “Thermodynamics of charged rotating black branes in Brans-Dicke theory with quadratic scalar field potential," Physical Review. D. Third Series, vol. 74, no. 10, Article ID 104014, 104014, 7 pages, 2006.

[14] S. H. Hendi, "Rotating black branes in Brans-Dicke-BORnInfeld theory," Journal of Mathematical Physics, vol. 49, no. 8, 082501, 12 pages, 2008.

[15] S. H. Hendi and R. Katebi, "Rotating black branes in BransDicke theory with a nonlinear electromagnetic field," European Physical Journal C, vol. 72, no. 11, article 2235, pp. 1-9, 2012.

[16] M. H. Dehghani and M. Shojania, "Travelling-wave-type and stationary soliton solutions of the Brans-Dicke equation," Canadian Journal of Physics, vol. 80, no. 9, pp. 951-958, 2002.

[17] S. H. Hendi and M. S. Talezadeh, "Nonlinearly charged dilatonic black holes and their Brans-Dicke counterpart: energy dependent spacetime," General Relativity and Gravitation, vol. 49, no. 1, Art. 12, 15 pages, 2017.

[18] G. Mie, "Grundlagen einer theorie der materie," Annals of Physics, vol. 37, no. 3, pp. 511-534, 1912.

[19] G. Mie, "Grundlagen einer theorie der materie zweite mitteilung," Annals of Physics, vol. 39, pp. 1-40, 1912.
[20] M. Born and L. Infeld, "Foundations of the new field theory," Proceedings of the Royal Society of London A, vol. 144, no. 852, pp. 425-451, 1934.

[21] E. S. Fradkin and A. A. Tseytlin, "Nonlinear electrodynamics from quantized strings," Physics Letters. B. Particle Physics, Nuclear Physics and Cosmology, vol. 163, no. 1-4, pp. 123-130, 1985.

[22] R. R. Metsaev, M. A. Rahmanov, and A. A. Tseytlin, "The BornInfeld action as the effective action in the open superstring theory," Physics Letters. B. Particle Physics, Nuclear Physics and Cosmology, vol. 193, no. 2-3, pp. 207-212, 1987.

[23] E. Bergshoeff, E. Sezgin, C. N. Pope, and P. K. Townsend, "The Born-Infeld action from conformal invariance of the open superstring," Physics Letters B, vol. 188, no. 1, pp. 70-74, 1987.

[24] C. G. Callan, C. Lovelace, C. R. Nappi, and S. . Yost, "Loop corrections to superstring equations of motion," Nuclear Physics. B. Theoretical, Phenomenological, and Experimental High Energy Physics. Quantum Field Theory and Statistical Systems, vol. 308, no. 2-3, pp. 221-284, 1988.

[25] O. D. Andreev and A. A. Tseytlin, "Partition-function representation for the open superstring effective action: cancellation of Möbius infinities and derivative corrections to Born-Infeld Lagrangian," Nuclear Physics. B. Theoretical, Phenomenological, and Experimental High Energy Physics. Quantum Field Theory and Statistical Systems, vol. 311, no. 1, pp. 205-252, 1988/89.

[26] R. Leigh, "Dirac-born-infeld action from dirichlet $\sigma$-model," Modern Physics Letters A, vol. 4, no. 28, article 2767, 1989.

[27] M. H. Dehghani, N. Alinejadi, and S. H. Hendi, “Topological black holes in Lovelock-Born-Infeld gravity," Physical Review D. Particles, Fields, Gravitation, and Cosmology, vol. 77, no. 10, Article ID 104025, 104025, 8 pages, 2008.

[28] M. H. Dehghani and S. H. Hendi, “Taub-NUT/bolt black holes in Gauss-Bonnet-Maxwell gravity," Physical Review. D. Third Series, vol. 73, no. 8, 084021, 11 pages, 2006.

[29] M. Allaverdizadeh, S. H. Hendi, J. P. Lemos, and A. Sheykhi, "Extremal Myers-Perry black holes coupled to Born-Infeld electrodynamics in odd dimensions," International Journal of Modern Physics. D. Gravitation, Astrophysics, Cosmology, vol. 23, no. 4, Article ID 1450032, 1450032, 13 pages, 2014.

[30] D. C. Zou, S. J. Zhang, and B. Wang, "Critical behavior of BornInfeld AdS black holes in the extended phase space thermodynamics," Physical Review D, vol. 89, Article ID 044002, 2014.

[31] R. Banerjee and D. Roychowdhury, "Critical behavior of BornInfeld AdS black holes in higher dimensions," Physical Review $D$, vol. 85, Article ID 104043, 2012.

[32] A. Lala and D. Roychowdhury, "Ehrenfest's scheme and thermodynamic geometry in Born-Infeld AdS black holes," Physical Review D-Particles, Fields, Gravitation and Cosmology, vol. 86, no. 8, Article ID 084027, 2012.

[33] R. Banerjee and D. Roychowdhury, "Critical phenomena in born-infeld AdS black holes," Physical Review D, vol. 85, Article ID 044040, 2012.

[34] P. Li, R.-H. Yue, and D.-C. Zou, "Thermodynamics of third order Lovelock-Born-Infeld black holes," Communications in Theoretical Physics, vol. 56, no. 5, pp. 845-850, 2011.

[35] D. Zou, Z. Yang, R. Yue, and P. Li, "Thermodynamics of GaussBonnet-BORn-Infeld black holes in AdS space," Modern Physics Letters A. Particles and Fields, Gravitation, Cosmology, Nuclear Physics, vol. 26, no. 7, pp. 515-529, 2011.

[36] A. Ghodsi and D. M. Yekta, "Black holes in Born-Infeld extended new massive gravity," Physical Review D - Particles, 
Fields, Gravitation and Cosmology, vol. 83, no. 10, Article ID 104004, 2011.

[37] R. G. Cai and Y. W. Sun, "Shear viscosity from AdS Born-Infeld black holes," Journal of High Energy Physics, vol. 9, article 115, 2008.

[38] S. H. Mazharimousavi, M. Halilsoy, and Z. Amirabi, "New nonabelian black hole solutions in Born-Infeld gravity," Physical Review D. Particles, Fields, Gravitation, and Cosmology, vol. 78, no. 6, Article ID 064050, 064050, 10 pages, 2008.

[39] W. A. Chemissany, M. de Roo, and S. Panda, "Thermodynamics of Born-Infeld black holes," Classical and Quantum Gravity, vol. 25, no. 22, Article ID 225009, 225009, 11 pages, 2008.

[40] Y. S. Myung, Y.-W. Kim, and Y.-J. Park, "Thermodynamics and phase transitions in the Born-Infeld-anti-de Sitter black holes," Physical Review D, vol. 78, no. 8, Article ID 084002, 2008.

[41] Y. S. Myung, Y.-W. Kim, and Y.-J. Park, "Thermodynamics of Einstein-Born-Infeld black holes in three dimensions," Physical Review D. Particles, Fields, Gravitation, and Cosmology, vol. 78, no. 4, 044020, 8 pages, 2008.

[42] O. Mišković and R. Olea, "Thermodynamics of Einstein-BornInfeld black holes with negative cosmological constant," Physical Review D, vol. 77, no. 12, 2008.

[43] I. Z. Stefanov, S. S. Yazadjiev, and M. D. Todorov, "Scalar-tensor black holes coupled to Born-Infeld nonlinear electrodynamics," Physical Review D. Particles, Fields, Gravitation, and Cosmology, vol. 75, no. 8, Article ID 084036, 084036, 6 pages, 2007.

[44] S. Fernando, "Thermodynamics of Born-Infeld-anti-de Sitter black holes in the grand canonical ensemble," Physical Review D, vol. 74, no. 10, Article ID 104032, 2006.

[45] R.-G. Cai, D.-W. Pang, and A. Wang, "Born-Infeld black holes in (A)dS spaces," Physical Review. D. Third Series, vol. 70, no. 12, Article ID 124034, 124034, 9 pages, 2004.

[46] M. a. Aiello, R. Ferraro, and G. Giribet, "Exact solutions of Lovelock-Born-Infeld black holes," Physical Review. D. Third Series, vol. 70, no. 10, Article ID 104014, 104014, 7 pages, 2004.

[47] T. Kumar Dey, "Born-Infeld black holes in the presence of a cosmological constant," Physics Letters. B. Particle Physics, Nuclear Physics and Cosmology, vol. 595, no. 1-4, pp. 484-490, 2004.

[48] T. Tamaki, "Black hole solutions coupled to Born-Infeld electrodynamics with derivative corrections," Journal of Cosmology and Astroparticle Physics, vol. 4, no. 5, article 004, 2004.

[49] S. Fernando and D. Krug, "Charged black hole solutions in Einstein-Born-Infeld gravity with a cosmological constant," General Relativity and Gravitation, vol. 35, no. 1, pp. 129-137, 2003.

[50] M. Wirschins, A. Sood, and J. Kunz, "Non-Abelian EinsteinBorn-Infeld black holes," Physical Review D, vol. 63, no. 8, 2001.

[51] M. Cataldo and A. García, "Three-dimensional black hole coupled to the Born-Infeld electrodynamics," Physics Letters. B. Particle Physics, Nuclear Physics and Cosmology, vol. 456, no. 1, pp. 28-33, 1999.

[52] M. H. Dehghani, S. H. Hendi, A. Sheykhi, and H. Rastegar Sedehi, "Thermodynamics of rotating black branes in Einstein-Born-Infeld-dilaton gravity," Journal of Cosmology and Astroparticle Physics, vol. 2, article 020, 2007.

[53] M. H. Dehghani and S. H. Hendi, "Thermodynamics of rotating black branes in Gauss-Bonnet-BORn-Infeld gravity," International Journal of Modern Physics. D. Gravitation, Astrophysics, Cosmology, vol. 16, no. 11, pp. 1829-1843, 2007.
[54] M. H. Dehghani and H. R. Rastegar Sedehi, “Thermodynamics of rotating black branes in $(n+1)$-dimensional Einstein-BornInfeld gravity," Physical Review. D. Third Series, vol. 74, no. 12, Article ID 124018, 124018, 6 pages, 2006.

[55] S. H. Hendi, "Rotating black string with nonlinear source," Physical Review D, vol. 82, no. 6, Article ID 064040, 2010.

[56] D. J. Cirilo Lombardo, "Rotating charged black holes in Einstein-Born-Infeld theories and their ADM mass," General Relativity and Gravitation, vol. 37, no. 5, pp. 847-856, 2005.

[57] V. Ferrari, L. Gualtieri, J. A. Pons, and A. Stavridis, "Rotational effects on the oscillation frequencies of newly born protoneutron stars," Monthly Notices of the Royal Astronomical Society, vol. 350, no. 2, pp. 763-768, 2004.

[58] H. Q. Lu, L. M. Shen, P. Ji, G. F. Ji, and N. . Sun, "Classical Euclidean wormhole solution and wave function for a nonlinear scalar field," International Journal of Theoretical Physics, vol. 42, no. 4, pp. 837-844, 2003.

[59] M. H. Dehghani and S. H. Hendi, "Wormhole solutions in Gauss-Bonnet-BORn-Infeld gravity," General Relativity and Gravitation, vol. 41, no. 8, pp. 1853-1863, 2009.

[60] E. F. Eiroa and G. Figueroa Aguirre, "Thin-shell wormholes with a generalized Chaplygin gas in Einstein-Born-Infeld theory," European Physical Journal C, vol. 72, no. 11, article 2240, pp. 1-6, 2012.

[61] S. H. Hendi, "Wormhole solutions in the presence of nonlinear maxwell field," Advances in High Energy Physics, vol. 2014, Article ID 697863, 11 pages, 2014.

[62] W. Yao and J. Jing, "Holographic entanglement entropy in insulator/superconductor transition with Born-Infeld electrodynamics," Journal of High Energy Physics, vol. 5, article 58, 2014.

[63] S. Gangopadhyay, "Holographic superconductors in Born-Infeld electrodynamics and external magnetic field," Modern Physics Letters A, vol. 29, no. 17, Article ID 1450088, 2014.

[64] S. Gangopadhyay, D. Roychowdhury, and JHEP., "Analytic study of Gauss-Bonnet holographic superconductors in Born-Infeld electrodynamics," Journal of High Energy Physics, vol. 5, article 156, 2012.

[65] S. Gangopadhyay, D. Roychowdhury, and JHEP., "Analytic study of properties of holographic superconductors in Born-Infeld electrodynamics," Journal of High Energy Physics, vol. 5, article 002, 2012.

[66] J. Jing, L. Wang, Q. Pan, and S. Chen, "Holographic superconductors in Gauss-Bonnet gravity with Born-Infeld electrodynamics," Physical Review D, vol. 83, no. 6, 2011.

[67] J. Jing and S. Chen, "Holographic superconductors in the Born-Infeld electrodynamics," Physical Letters B, vol. 686, no. 1, pp. 68-71, 2010.

[68] S. H. Hendi, M. R. Hadizadeh, and R. Katebi, "Combination of born-infeld and conformally invariant maxwell lagrangians," Iranian Journal of Science and Technology, Transactions A: Science, pp. 1-10, 2016.

[69] B. Vaseghi, G. Rezaei, S. H. Hendi, and M. Tabatabaei, "Comparison between the nonlinear born-infeld and coulomb theories in quantum dots," Quantum Matter, vol. 2, pp. 194-198, 2013.

[70] S. W. Hawking, "Black hole explosions?" Nature, vol. 248, no. 5443, pp. 30-31, 1974.

[71] J. D. Bekenstein, "Black holes and entropy," Physical Review D, vol. 7, pp. 2333-2346, 1973.

[72] S. W. Hawking and D. N. Page, "Thermodynamics of black holes in anti-de Sitter space," Communications in Mathematical Physics, vol. 87, no. 4, pp. 577-588, 1982/83. 
[73] R.-G. Cai, S. P. Kim, and B. Wang, "Ricci flat black holes and Hawking-Page phase transition in Gauss-Bonnet gravity and dilaton gravity," Physical Review D. Particles, Fields, Gravitation, and Cosmology, vol. 76, no. 2, Article ID 024011, 2007.

[74] R. G. Cai, L. M. Cao, and Y. W. Sun, "Hawking-Page phase transition of black Dp-branes and R-charged black holes with an IR cutoff," Journal of High Energy Physics, vol. 11, article 039, 2007.

[75] M. Eune, W. Kim, and S. H. Yi, "Hawking-Page phase transition in BTZ black hole revisited," Journal of High Energy Physics, vol. 3, article 20, 2013.

[76] P. C. W. Davies, “The thermodynamic theory of black holes,” vol. 353, no. 1675, pp. 499-521, 1977.

[77] H. B. Callen, Thermodynamics and an Introduction to Thermostatistics, Wiley, New York, NY, USA, 1985.

[78] F. Weinhold, "Metric geometry of equilibrium thermodynamics," The Journal of Chemical Physics, vol. 63, no. 6, pp. 24792483, 1975.

[79] F. Weinhold, "Metric geometry of equilibrium thermodynamics. II. Scaling, homogeneity, and generalized Gibbs-Duhem relations," The Journal of Chemical Physics, vol. 63, no. 6, pp. 2484-2487, 1975.

[80] G. Ruppeiner, "Thermodynamics: a Riemannian geometric model," Physical Review A, vol. 20, no. 4, pp. 1608-1613, 1979.

[81] G. Ruppeiner, "Riemannian geometry in thermodynamic fluctuation theory," Reviews of Modern Physics, vol. 67, no. 3, pp. 605-659, 1995.

[82] P. Salamon, J. Nulton, and E. Ihrig, "On the relation between entropy and energy versions of thermodynamic length," The Journal of Chemical Physics, vol. 80, no. 1, pp. 436-437, 1984.

[83] H. Quevedo, "Geometrothermodynamics," Journal of Mathematical Physics, vol. 48, no. 1, Article ID 013506, 013506, 14 pages, 2007.

[84] H. Quevedo and A. Sanchez, "Geometrothermodynamics of asymptotically anti-de Sitter black holes," Journal of High Energy Physics, vol. 9, article 034, 2008.

[85] S. H. Hendi, S. Panahiyan, B. Eslam Panah, and M. Momennia, "A new approach toward geometrical concept of black hole thermodynamics," The European Physical Journal C, vol. 75, no. 10, article 507, 2015.

[86] S. H. Hendi, S. Panahiyan, and B. Eslam Panah, "Geometrical method for thermal instability of nonlinearly charged BTZ black holes," Advances in High Energy Physics, vol. 2015, Article ID 743086, 2015.

[87] S. H. Hendi, A. Sheykhi, S. Panahiyan, and B. Eslam Panah, "Phase transition and thermodynamic geometry of EinsteinMaxwell-dilaton black holes," Physical Review D. Particles, Fields, Gravitation, and Cosmology, vol. 92, no. 6, Article ID 064028, 064028, 10 pages, 2015.

[88] S. H. Hendi, R. M. Tad, Z. Armanfard, and M. S. Talezadeh, "Extended phase space thermodynamics and $\mathrm{P}-\mathrm{V}$ criticality: Brans-Dicke-Born-Infeld vs. Einstein-Born-Infelddilaton black holes," European Physical Journal C, vol. 76, no. 5, article 263, 2016.

[89] A. Sheykhi, "Thermodynamics of charged topological dilaton black holes," Physical Review D. Particles, Fields, Gravitation, and Cosmology, vol. 76, no. 12, Article ID 124025, 124025, 8 pages, 2007.

[90] C. Niu, Y. Tian, and X. N. Wu, "Critical phenomena and thermodynamic geometry of Reissner-Nordström-anti-de Sitter black holes," Physical Review D, vol. 85, no. 2, Article ID 024017, 8 pages, 2012.
[91] S. H. Hendi, S. Panahiyan, B. E. Panah, and Z. Armanfard, "Phase transition of charged Black Holes in Brans-Dicke theory through geometrical thermodynamics," European Physical Journal C, vol. 76, no. 7, article 396, 2016.

[92] G. Amelino-Camelia, "Relativity in spacetimes with shortdistance structure governed by an observer-independent (Planckian) length scale," International Journal of Modern Physics. D. Gravitation, Astrophysics, Cosmology, vol. 11, no. 1, pp. 35-59, 2002.

[93] G. Amelino-Camelia, "Phenomenology of Planck-scale Lorentz-symmetry test theories," New Journal of Physics, vol. 6, p. 188, 2004.

[94] J. Magueijo and L. Smolin, "Gravity's rainbow," Classical and Quantum Gravity, vol. 21, no. 7, pp. 1725-1736, 2004.

[95] S. H. Hendi, M. Momennia, B. E. Panah, and M. Faizal, "Nonsingular universes in gauss-bonnet gravity's rainbow," Astrophysical Journal, vol. 827, no. 2, article 153, 2016. 

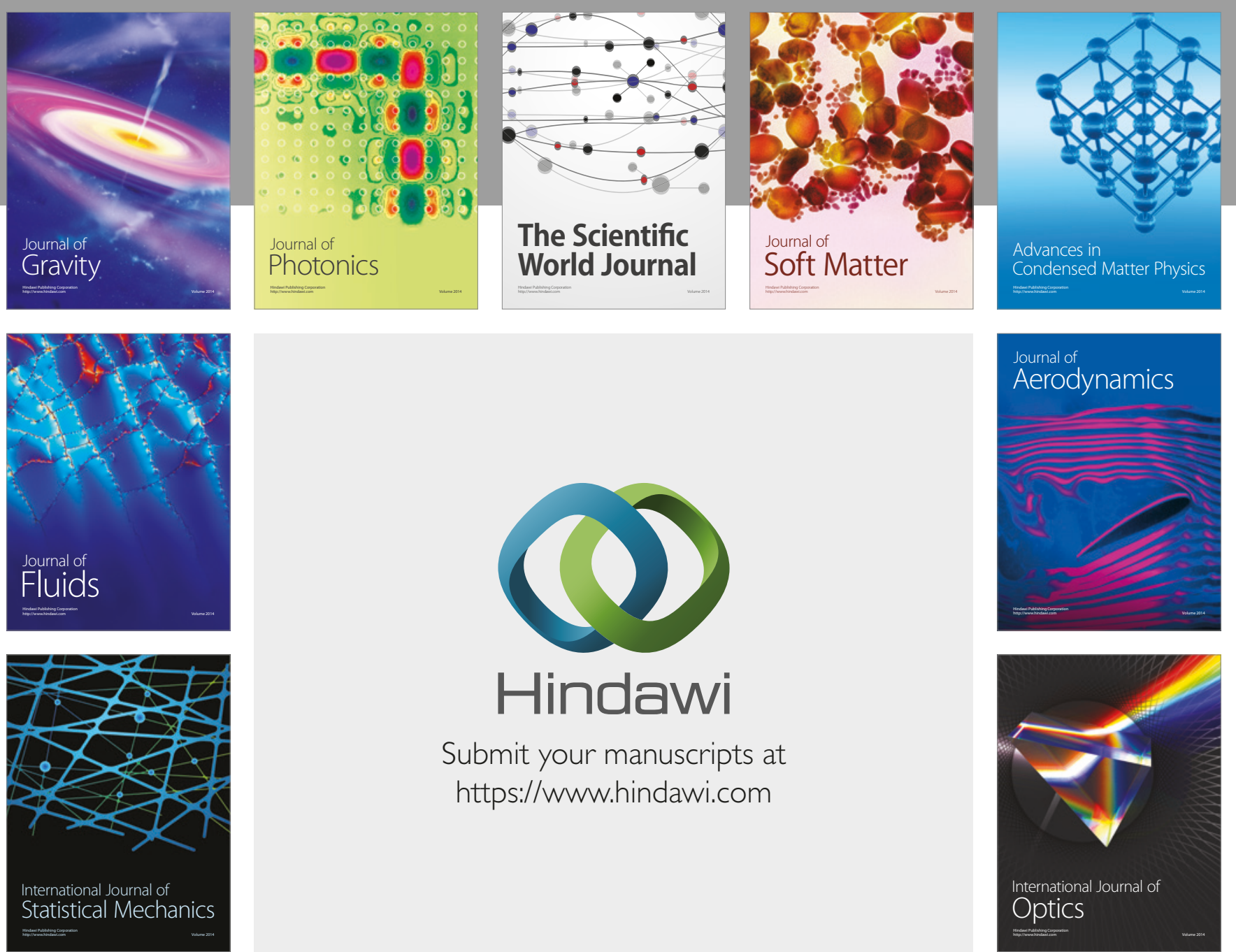

Submit your manuscripts at

https://www.hindawi.com
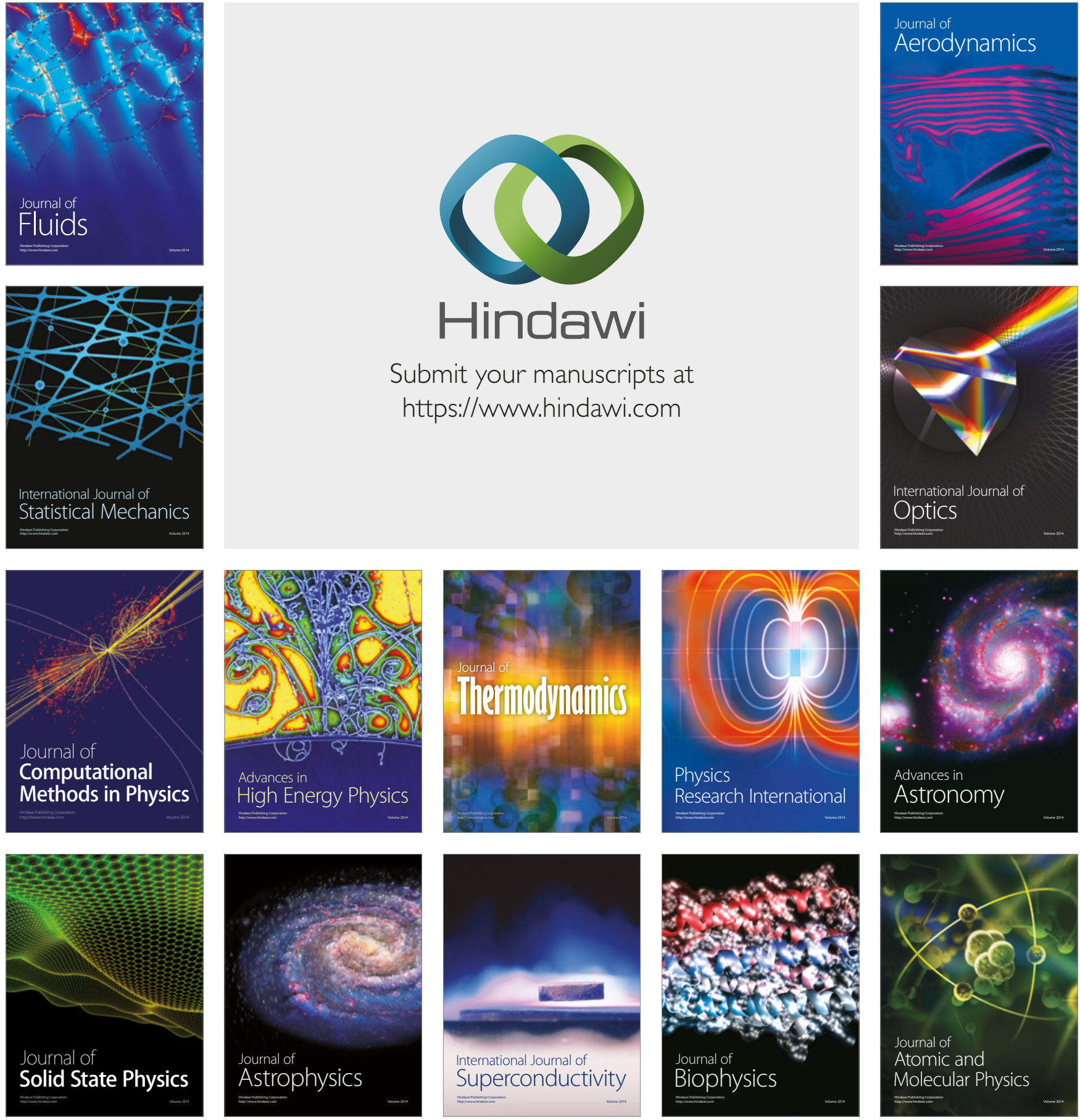